\title{
Spin-Orbit Coupling of Light in Photonic Crystal Waveguides
}

\author{
Moïse Sotto \\ Sustainable Electronic Technologies, Electronics and Computer Science, \\ Faculty of Physical Science and Engineering, University of Southampton, \\ 8 University Road, Southampton, SO17 1BJ, UK. \\ Kapil Debnath \\ Department of Electronics 83 Electrical Communication Engineering, \\ Indian Institute of Technology Kharagpur, Kharagpur, West Bengal 721302, India. \\ Isao Tomita \\ Department of Electrical \& Computer Engineering, National Institute of Technology, \\ Gifu College, 2236-2 Kamimakuwa, Motosu-city, Gifu 501-0495, Japan \\ Shinichi Saito* \\ Sustainable Electronic Technologies, Electronics and Computer Science, \\ Faculty of Physical Science and Engineering, University of Southampton, \\ 8 University Road, Southampton, SO17 1BJ, UK.
}

(Dated: December 20, 2018)

\begin{abstract}
We investigate the effect of breaking the parity of a photonic crystal waveguide designed to have odd and even modes intersecting inside the photonic bandgap. The complete study on the wavefields properties of the resulting bonding modes uncovers that the transverse spin and orbital parts of the angular momentum couple in the very slow-light regime by the means of their local currents. This leads to a sub-bandgap opening between bands characterized by distinct organizations of this spinorbit coupling at the nano-scale.
\end{abstract}

\section{INTRODUCTION}

Photonic Crystals (PhCs) are the optical analogues of atomic crystal lattices in which dielectric compounds are periodically arranged to shape the propagation of photons $[1,2]$. In the case where the periodicity and the dielectric contrast are suitably set, complete energy gaps emerge and transform the $\mathrm{PhC}$ into a semiconductor for light [3-5]. Albeit the prodigious confinement properties brought by such a photonic bandgap in itself have important implications for light circuitry on-chip, like the slow-light regime[6-9] or the Purcell effect[10-12] which have been investigated for three decades now, the strong confinement of light provided by $\mathrm{PhC}$ has more to offer. Cleverly designed structured photonic networks can trigger Angular Momentum (AM) degrees of freedom like their electronic counterparts: the Spin Angular Momentum (SAM) and the Orbital Angular Momentum (OAM). The SAM is intrinsic and corresponds to the circular polarization with left or right handedness while the OAM in its intrinsic form appears as an optical vortex characterized by its topological charge[13? -15]. 2D PhC hole slabs are well known to support a Transverse Electric (TE) photonic bandgap, thus photonic crystal waveguide (PCW) modes guided by this bandgap mechanism have a non-paraxial propagation. In other words, the electric field $\vec{E}$ of confined mode has both transverse and

\footnotetext{
* S.Saito@soton.ac.uk
}

longitudinal components $\mathrm{E}_{y}$ and $\mathrm{E}_{x}$ respectively. In the case where these two components are in quadrature, the electric field is circularly polarized in a plane containing the propagation direction[16, 17]. Accordingly, such circularly polarized points (C-points) are labeled as transverse SAM and are sometimes referred to in the literature as photonic wheels[18-22], which starkly contrasts with the familiar case of an electric field spinning around the propagation axis. The orbital degree of freedom can $a$ fortiori be transverse, because it derives from the phase structure of the light. In order to be considered as photonic wheels, the vortices holding the OAM must wind around an axis perpendicular to the propagation[15, 23].

Due to the spatial confinement of light in nano-photonic devices and its evanescent nature in the vicinity of interfaces, numerous applications on-chip are promising for both transverse OAM and SAM ranging from plasmonics to atomtronics as well as Silicon ( $\mathrm{Si}$ ) photonics. Topological action is expected to be heralded by the transverse aspect of these AM, thus forcing the clockwise and the anti-clockwise rotation to be associated with opposite directions of propagation. It has already been demonstrated that photonic wheels are able to directionally excite surface-plasmon-polaritons at a metal-dielectric interface [24], waveguide modes at the output of a subwavelength microdisk resonator[25], Zeeman sub-level of a single ultra-cold atom[26] and even PCW modes through dipolar transition of quantum dots[27, 28]. In all the examples listed, the chiral behavior derives from a broken symmetry in the system, which limits directional routing in standard PCWs belonging to the symmorphic space 
group. Locally, AM are extremely sensitive to the symmetries, especially in inhomogeneous media[29, 30] like PhCs. Thus the break of important symmetries like rotational[31, 32] or inversion symmetry[33, 34] opens a non-trivial gap between bands characterized by different Chern numbers[35, 36]. Symmorphic PCWs confine pair-wise modes with both odd and even symmetries that can intersect without interacting with each other due to their different parities $[9,37]$. Breaking the parity by substituting the mirror symmetry with a glideplane symmetry allows a branch exchange between these modes raising the degeneracy[38-40], which is somehow reminiscent of the spin-orbit coupling effect on energy bands in atomic crystals[41]. Inducing spin and orbital fluxes in photonic systems is a crucial step towards the realization of directional routing for light circuitry onchip and hence architect scalable photonic networks with logic gates able to deliver different outputs distinct by their directionality. It is the purpose of this work to understand the nature of spin and orbital currents in the anomalous slow-light regime of a photonic bandgap, the interplay between OAM and SAM at the nano-scale and study how these wavefield properties can be engineered through modification of the planar structure in order to possibly functionalize them as the encoding mechanism of the next-generation of optical communication protocols.

Here, we present a complete study on the anti-crossing taking place when the parity of a symmorphic PCW is broken and its impact on both the spin and orbital AM local currents in the particular context of Zero-Group Velocity (ZGV) $[15,42,43]$. A clear picture of the complete wavefields is obtained by using the three-dimensional Finite-Difference-Time-Domain (3D-FDTD) method and leads to the main result: a gap opens between bands that encapsulate different organizations of the spin-orbit coupling at the nano-scale. In section II, we emphasize the consequence of breaking the parity on the photonic bandstructure and point out how it gives rise to the formation of hybrid modes neither odd nor even accompanied by the emergence of the slow-light regime at anomalous position in the Brillouin Zone (BZ). Section III presents the properties of the cycle-averaged electromagnetic fields in the plane of symmetry $(\mathrm{z}=0)$ of the PCW. The transverse SAM and OAM are clearly identified by the means of the Stokes parameters and the analysis of the modal phase structure respectively. The analysis of the electromagnetic fields unveils that photonic wheels do not necessarily vanish as the light slows down. Furthermore, the influence of the optical vortices on the state of polarization is underlined by examining its evolution on the Poincaré sphere and reveals that the OAM acts as an effective polarization for the envelop streamlines. Finally, in Section IV we move from the previously used two-dimensional description of the modal properties to a three-dimensional analysis of the SAM and OAM densities with the appropriate Minkowski formalism[29, 30, 44]. These quantities, representing the local fluxes of the respective AM,

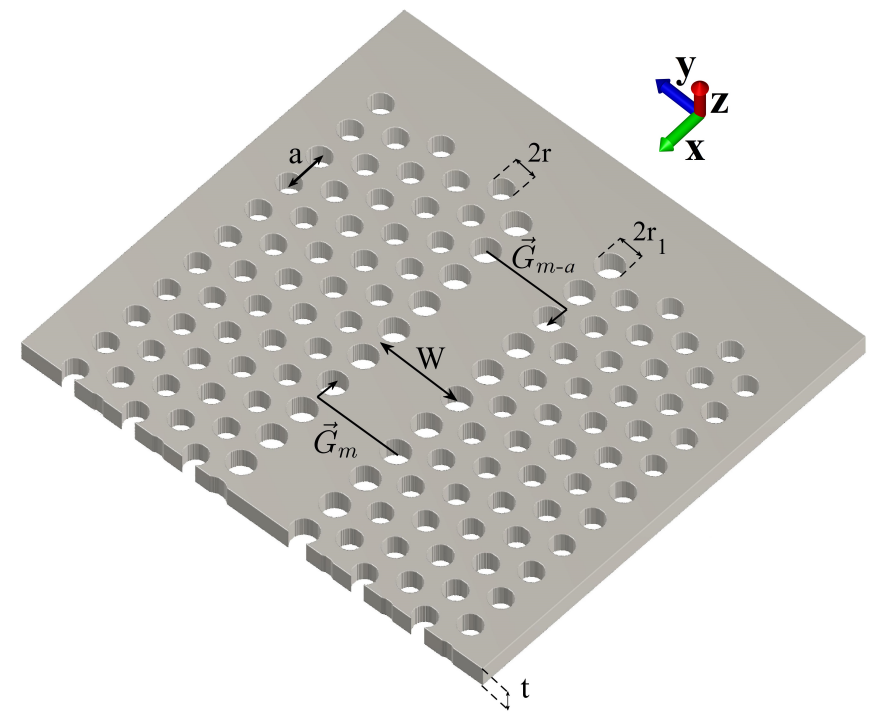

FIG. 1. The PhC is an hexagonal lattice of period $a=0.415 \mathrm{~nm}$ patterned with holes of radii $r=0.3 a$ in a Si slab of thickness $\mathrm{t}=220 \mathrm{~nm}$. The waveguide has a width $\mathrm{W}=1.3 \sqrt{3} a$ and the first rows of holes have a modified radius of $r_{1}=0.35 a$. One side of the $\mathrm{PhC}$ is shifted along the propagation direction by an offset $m$ with respect to the other, such as the mirror symmetry is replaced by the glide-plane axis $\overrightarrow{\mathrm{G}}_{m}$ and $\overrightarrow{\mathrm{G}}_{m-a}$.

provide a deeper insight into the structure of the light and allow one to understand how the non-paraxial guidance and the slow-down effect, induced by the photonic bandgap, organize the SAM(OAM) by the means of the $\mathrm{OAM}(\mathrm{SAM})$ density. These features represent a clear manifestation of the spin-orbit coupling in this PCW occurring at subwavelength dimensions. Outlook about the potential applications of controlling the SAM and OAM of light at the nano-scale is given in section IV to conclude the paper.

\section{BANDSTRUCTURE: THE EFFECT OF BREAKING THE PARITY SYMMETRY}

Recently, the tight confinement offered by 2D PhCs and the resulting non-paraxial guidance of light enabled the manipulation of the directional emission of an embedded quantum emitter by engineering the density of photonic states at C-points[27]. This effect is salient in non-symmorphic PCWs[39] where the mirror symmetry of the structure is replaced by glide-plane symmetries. To qualitatively explain why, we propose to express the field intensity at the center of a symmorphic PCW, in terms of Left Circularly Polarized (LCP) and Right Circularly Polarized (RCP) vectors $\left(\overrightarrow{\mathrm{E}}_{\wp} / \overrightarrow{\mathrm{E}}_{\mathrm{C}}=\left(\mathrm{E}_{x} \vec{x} \pm i \mathrm{E}_{y} \vec{y}\right) / \sqrt{2}\right)$. The longitudinal and transverse electric components of a TE guided mode have opposite parities given by the 
relations:

$$
\begin{aligned}
& \mathrm{E}_{x}(x, y, z)= \pm \mathrm{E}_{x}(x,-y, z), \\
& \mathrm{E}_{y}(x, y, z)=\mp \mathrm{E}_{y}(x,-y, z),
\end{aligned}
$$

where the mode parity is classified with respect to the transverse component. It follows that the RCP and LCP components have the same norm and a phase difference $\delta=0$ or $\pm \pi$ at $y=0$, where the electric field maximum is located. Thus, annihilating the ellipticity of the light in the middle of the PCW.

On the contrary, breaking the PCW parity makes the parity classification of Eq.(1) obsolete[39], thus allowing chiral and high field intensity regions to overlap. The design parameters used to form these hybrid bands are represented in Fig.1. The $1.3 \sqrt{3} a$ wide PCWs ( $y$ component of center-to-center distance between two holes bordering the waveguide) are formed in a hexagonal lattice of period $a=415 \mathrm{~nm}$ patterned with holes of radii $r=0.3 a$ in a $220 \mathrm{~nm}$ thick Si slab, the radii of the first rows of holes are extended to $r_{1}=0.35 a$. One side of the $\mathrm{PhC}$ is shifted, with respect to the other, by an offset $m$ along the propagation direction. In this instance, the mirror symmetry operations Eq.(1) are replaced by the glide-plane transformations as follows:

$$
\begin{array}{r}
\hat{\mathrm{G}}_{m} \overrightarrow{\mathrm{E}}(x, y, z)=e^{i m k} \overrightarrow{\mathrm{E}}(x,-y, z), \\
\hat{\mathrm{G}}_{m-a} \overrightarrow{\mathrm{E}}(x,-y, z)=e^{i(m-a) k} \overrightarrow{\mathrm{E}}(x, y, z),
\end{array}
$$

where $\hat{\mathrm{G}}_{m}$ is the glide-plane operator and $y>0$.

The transformation of the bandstructure, calculated by the 3D-FDTD method, as $m$ is scanned from 0 to $a / 2$ is shown in Fig.2. Modes originally crossing, due to their different parities under mirror symmetry, bond together

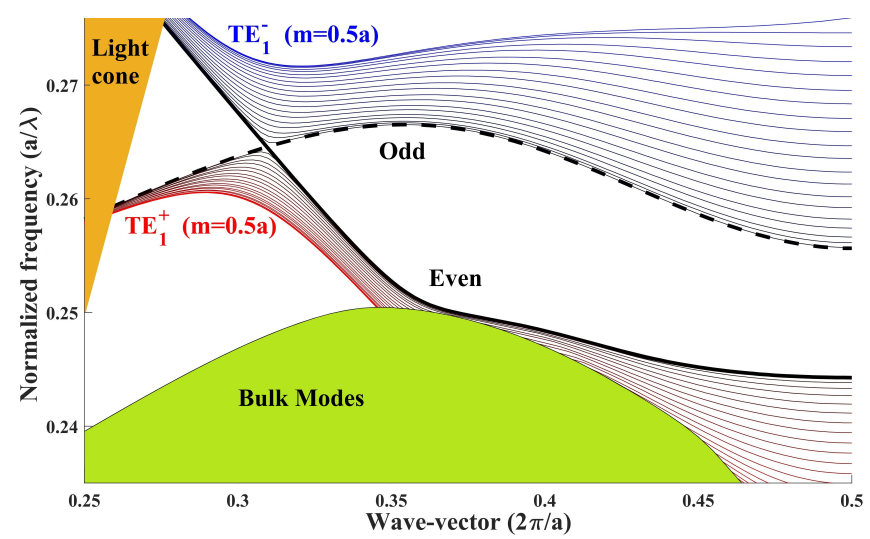

FIG. 2. Bandstructures of the PCW for different offset $m$ between the two side of the $\mathrm{PhC}$. The $\mathrm{PhC}$ bulk modes are represented in green and the light-cone in orange. The dispersion relation for the symmorphic structure $(\mathrm{m}=0)$ is represented in dashed and full black lines for the odd and even modes respectively. The offset couples odd and even which gives rise to the $\mathrm{TE}_{1}^{+}$and $\mathrm{TE}_{1}^{-}$modes in shades of red and blue respectively. These modes are represented in red and blue for the maximum offset $m=0.5 a$. and exchange their branches, thus allowing ZGV points to appear far from the edge of the BZ. This anomalous position for the slow-light implies that geometrical phases persist in the fields patterns (i.e. $k a \neq \pi$ ) and the energy flux represented by the Poynting vector:

$$
\overrightarrow{\mathcal{P}}=\frac{1}{2} \operatorname{Re}\left(\overrightarrow{\mathrm{E}}^{*} \times \overrightarrow{\mathrm{B}}\right)
$$

cannot simply vanish everywhere as it does at the bandedge. On the other hand, the total energy flow passing through the unit-cell must be null in order to also satisfy its scalability with the group velocity.

\section{ELECTROMAGNETIC WAVE PROPERTIES}

Here, the properties of the electromagnetic fields are presented at the crossing point for the even and odd modes and at the anomalous ZGV points for the $\mathrm{TE}_{1}^{+}$ and $\mathrm{TE}_{1}^{-}$modes. From now, the even and odd $\left(\mathrm{TE}_{1}^{+}\right.$ and $\mathrm{TE}_{1}^{-}$) modes are referred to as the crossing (aZGV) point without any ambiguity. The electric and magnetic fields profiles are distinctively described in the plane of symmetry to emphasize that they hold separate parts of the AM. Nevertheless, a first indication of the interplay between the spin and orbital AM in both the bonding modes is presented through the energy streamlines.

\section{A. Electric fields properties: Stokes Parameters, Poincaré Sphere and SAM.}

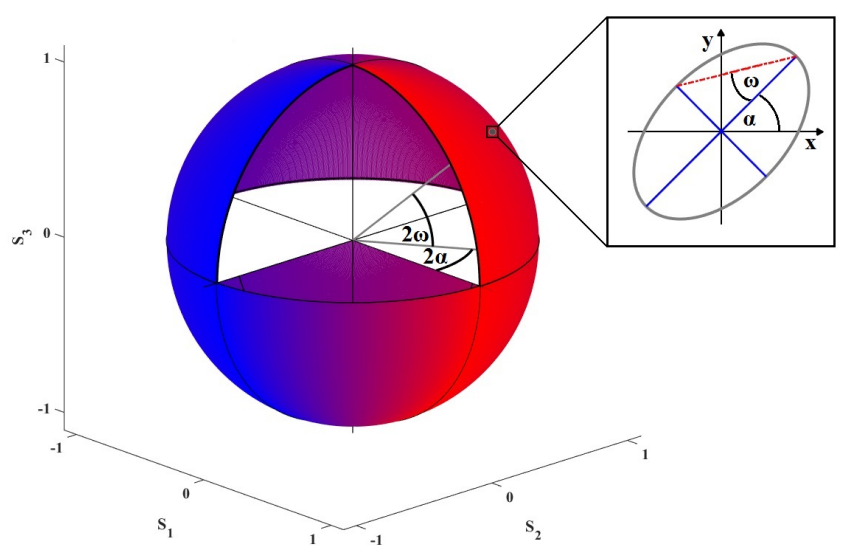

FIG. 3. The Poincaré sphere can be represented with the Stokes parameters $S_{1}, S_{2}$ and $S_{3}$ in the Cartesian coordinate system or in the spherical coordinate system with the angles $\alpha$ and $\omega$, parametrizing the polarization ellipse (inset), times a factor 2 .

Despite the non-paraxial guidance of light in PCWs, it can be considered that light is completely polarized in the plane of symmetry $(\mathrm{z}=0)[45]$, because guided modes 


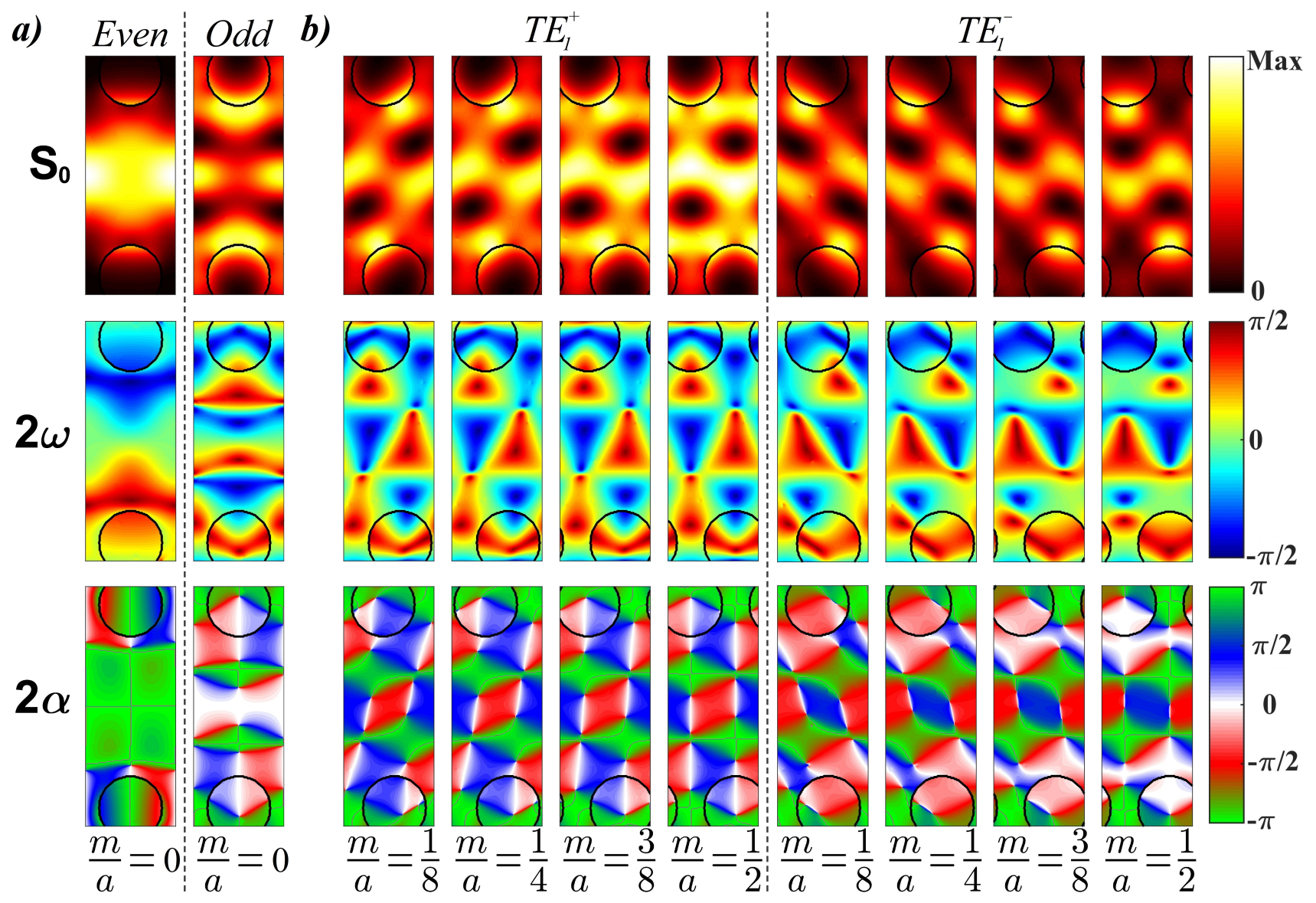

FIG. 4. Polarization characteristics of the pair-wise modes in the symmorphic structure (a) and of the $\mathrm{TE}_{1}^{+}$and $\mathrm{TE}_{1}^{-}$modes for different $m(\mathrm{~b})$. The top panels represent the Stokes parameter $S_{0}$ which is normalized with respect to the highest field intensity in each graph. The middle and bottom panels describe the angles $2 \omega$ and $2 \alpha$ within the PCW unit-cell respectively.

have there orthogonal electric and magnetic fields. For instance, the fields components of a TE mode are expressed by:

$$
\begin{array}{r}
\overrightarrow{\mathrm{E}}(x, y, z=0)=\left(\mathrm{E}_{x}, \mathrm{E}_{y}, 0\right), \\
\overrightarrow{\mathrm{H}}(x, y, z=0)=\left(0,0, \mathrm{H}_{z}\right) .
\end{array}
$$

Therefore, the polarization can be characterized in this plane by using the Stokes parameters:

$$
\begin{aligned}
& S_{0}=\left|\mathrm{E}_{x}\right|^{2}+\left|\mathrm{E}_{y}\right|^{2}, \\
& S_{1}=\left|\mathrm{E}_{x}\right|^{2}-\left|\mathrm{E}_{y}\right|^{2}=S_{0} \cos (2 \omega) \cos (2 \alpha), \\
& S_{2}=2 \operatorname{Re}\left(\mathrm{E}_{x}^{*} \mathrm{E}_{y}\right)=S_{0} \cos (2 \omega) \sin (2 \alpha), \\
& S_{3}=2 \operatorname{Im}\left(\mathrm{E}_{x}^{*} \mathrm{E}_{y}\right)=S_{0} \sin (2 \omega),
\end{aligned}
$$

which respect the full polarization condition[46]:

$$
\sum_{i=1,2,3} S_{i}^{2}(x, y, 0)=S_{0}^{2}(x, y, 0) .
$$

The Stokes parameters $S_{1}, S_{2}$ and $S_{3}$ refer to the description of the polarization in the linear $\left(\mathrm{E}_{x}, \mathrm{E}_{y}\right)$, diag- onal $\left(\mathrm{E}_{x}+\mathrm{E}_{y}, \mathrm{E}_{x}-\mathrm{E}_{y}\right) / \sqrt{2}$ and circular $\left(\mathrm{E}_{Ð}, \mathrm{E}_{\mathrm{C}}\right)$ polarization bases respectively. Their complementarity allows the representation of the state of polarization as a point on the surface of the Poincaré sphere[13, 14, 46] as shown in Fig.3, which is also defined by the azimuthal and polar angles $2 \alpha$ and $2 \omega$ introduced in the third set of Eq.(5). These angles also characterize the polarization ellipse times a factor 1/2 (see Fig.3 inset). This factor comes from the fact that the rotation of the polarization state on the surface of the Poincaré sphere has an $\mathrm{SO}(3)$ representation, while the polarization ellipse can be transformed through the Jones calculus equivalent to $\mathrm{SU}(2)$ transformations and typical of a spin-half system. Nevertheless, due the longitudinal component of $\overrightarrow{\mathrm{E}}$, both RCP and LCP spin around the $z$-axis, which classifies the SAM in our system as transverse.

Figure 4 displays the spatial dependence of $S_{0}, 2 \alpha$ and $2 \omega$ in the PCW unit-cell for odd and even modes at the crossing points as well as for $\mathrm{TE}_{1}^{+}$and $\mathrm{TE}_{1}^{-}$modes with different $m$. The RCP(LCP) are depicted by $2 \omega= \pm \pi / 2$ but have an undefined $2 \alpha$. It can be seen in the relevant figures that at these locations, all the $2 \alpha$ values are merging towards the C-points. Since the electric fields of the 


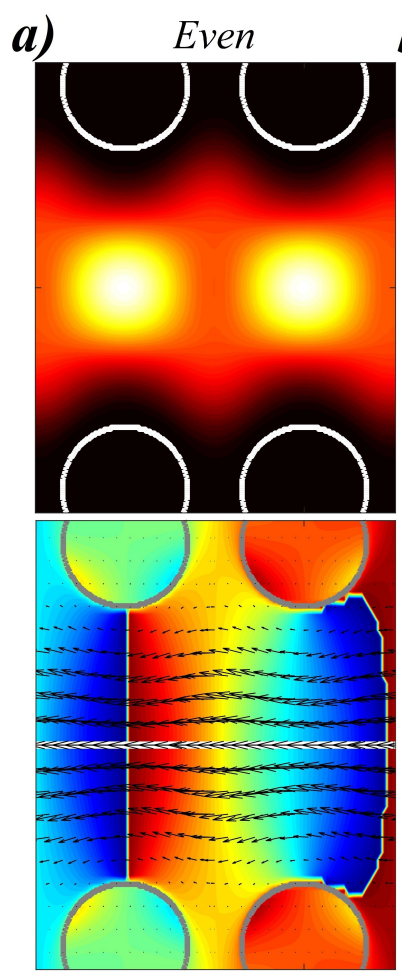

b)
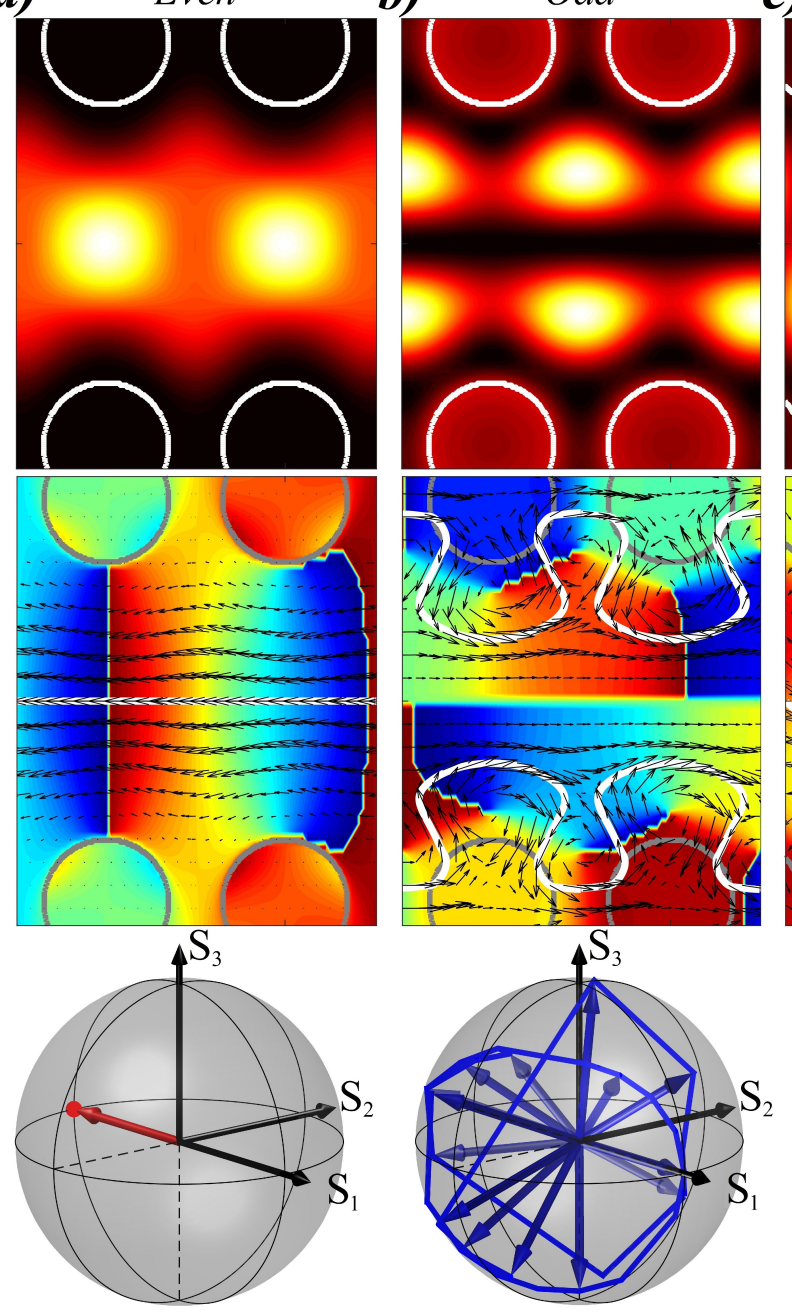

c)
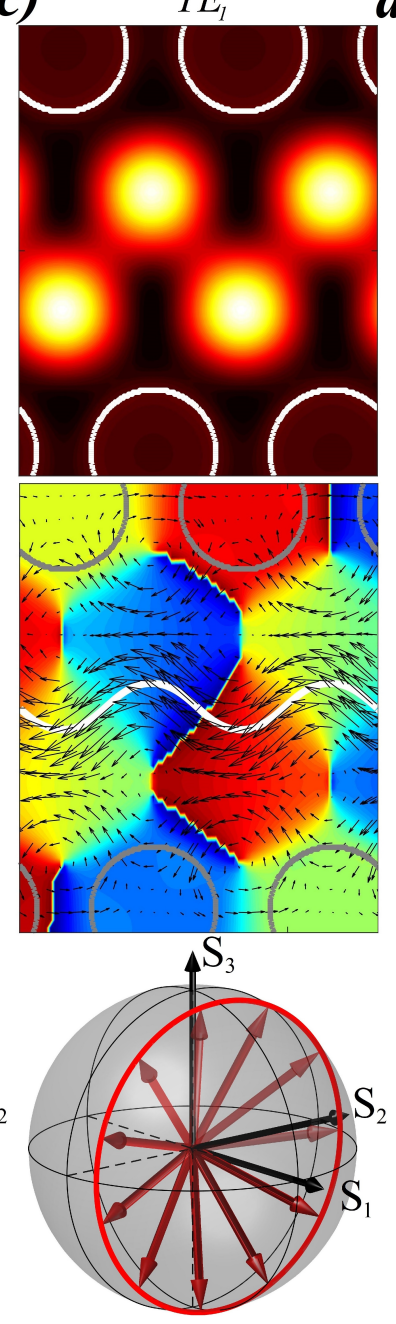

d)
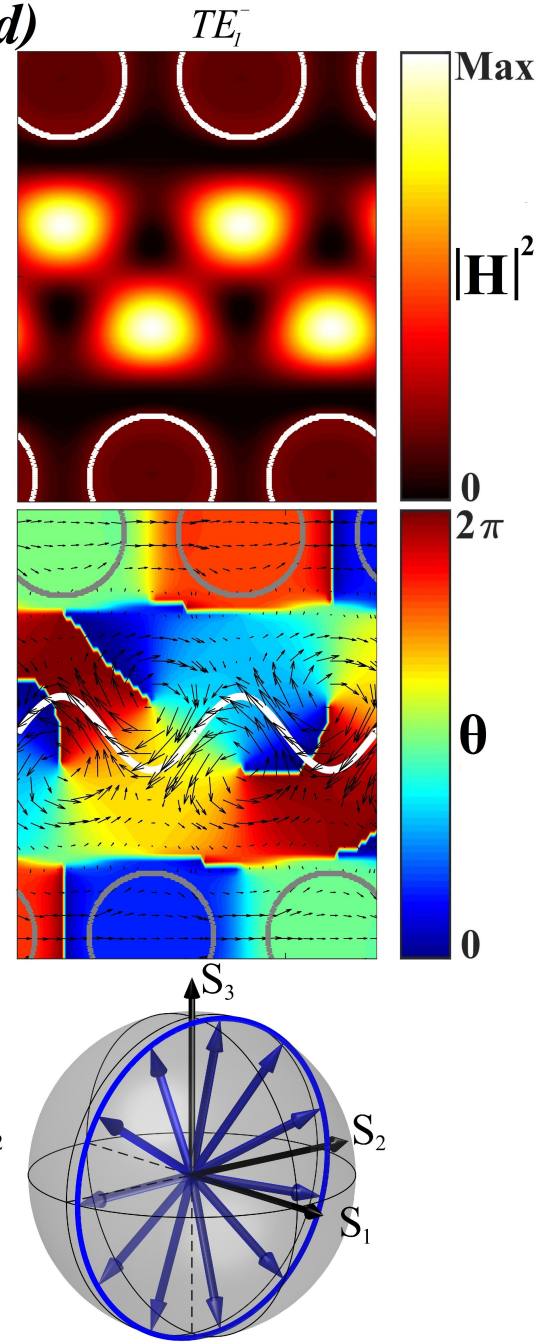

FIG. 5. Magnetic Field properties of the even (a), odd (b), $\mathrm{TE}_{1}^{+}$(c) and $\mathrm{TE}_{1}^{-}$(d) modes in the plane of symmetry of the PCWs. The top panels represent the magnetic field intensity of the modes $|H|^{2}$. On the middle panels, the phase patterns $\theta$ of the modes are represented by the contour plots on which the black arrows, corresponding to the Poynting vector $\overrightarrow{\mathcal{P}}$, have been superimposed. The white line is the major streamline. The polarization along these streamlines has been plotted on the Poincaré sphere in the bottom panel of the relevant figures. The opacity of the polarization vector increases along the propagation. For simplicity, only the streamline in the upper part of the PCW is represented for the odd mode (b).

even and odd modes are mostly linearly polarized along the transverse and longitudinal direction respectively, Cpoints do not coincide with high intensity regions in the symmorphic configuration, as shown in Fig.4(a). This difference in their polarization arises from their distinct guidance mechanisms. The even mode is index-guided and experiences the $\mathrm{PhC}$ as a periodic corrugation, its confinement relies only on the total-internal reflection. On the other hand, the odd mode is guided by the photonic bandgap due its penetration into the $\mathrm{PhC}$ bulks. The Bragg mechanism enforces this mode to have its dominant electric field component parallel to the direction of propagation.

Nevertheless, the situation is rather different once the parity symmetry is broken, as shown in Fig. 4(b). The branch exchange between these modes with orthogonal dominant polarizations results in bonding modes not only supporting C-points at high field intensity regions but also providing a degree of ellipticity to the electric field maxima. Here, the interest comes from the fact that the SAM arises despite the ZGV, while in standard PCWs, if the band-edge is generally the region of the slow-light effect, the matching of the wave-vector with the periodicity annihilates the chirality because the associated fields become purely real:

$$
\lim _{k \rightarrow \frac{\pi}{a}} \operatorname{Im}(\overrightarrow{\mathrm{E}}) \rightarrow \overrightarrow{0}
$$

Conversely, modes guided by wave-vectors different from high symmetry points of the BZ must be associated with fields having a non-zero imaginary part because the translation of a period induces a phase shift $\neq 0$ or $\pm \pi$. 
In our case, it can be concluded that breaking the parity symmetry is beneficial to form hybrid modes holding enhanced transverse SAM at anomalous ZGV points, showing that photonic wheels do not always stop turning when the light slow down.

\section{B. Magnetic fields properties: vortices, topological charge and OAM}

In the former part, it has been shown that the electric fields of the bonding modes carry the transverse SAM degree of freedom, which is conserved by the periodicity (translation by a period) and reversed by the glide-plane axes $\vec{G}_{m}$ and $\vec{G}_{m-a}$. Therefore, it is natural to examine the properties of the magnetic field in the plane of symmetry and seek the presence of an other AM: the orbital degree of freedom. For simplicity, only the maximum offset $m / a=1 / 2$ will be explored from now on.

Prior to the description of the bonding modes properties, the magnetic fields of the even and odd modes are analyzed. The intensity as well as the phase:

$$
\theta=\arg \left(\mathrm{H}_{z}\right)
$$

profiles of these modes are reported in Fig.5(a)-(b) in the top and middle panels respectively. In order to study the impact of the guidance mechanism on the field properties, $\overrightarrow{\mathcal{P}}$ is superimposed onto the phase pattern of the mode. As seen previously, the even mode does not differ fundamentally from a single mode in a normal ridge Si waveguide, except its backward propagation due to the periodicity. All the energy flows are confined within the core of the waveguide, a region that was previously characterized as polarized along $\vec{y}$ (Fig.4(a)). The polarization along the major streamline of $\overrightarrow{\mathcal{P}}$ is reported in the bottom panel of Fig.5(a) and clearly shows that the light flows without changing its polarization.

On the contrary, the odd mode, shown in Fig.5(b), is guided by the photonic bandgap and presents a group index an order of magnitude higher than the even mode. The intensity pattern shows that the magnetic field is not only confined in the central region but also extends into the PhC bulks. Due to the higher order of this mode, one can distinguish a few lobes with nodes between them. Its phase pattern has a strong modulation with a patchy structure and highlights that the aforementioned nodes are the centers of optical vortices. The phase is undetermined in the center of the vortices and jumps by $\pm 2 \pi$ around them such as:

$$
l=\frac{1}{2 \pi} \int_{L} \vec{\nabla}(\theta) \cdot d \vec{s}= \pm 1,
$$

is the topological charge of these vortices, where $\mathrm{L}$ is a closed contour around a vortex. The energy flow is circulating around the phase singularities and propagates by undulating between vortices with opposite topological charges, which explains the origin of the slow-down effect for this mode. However, the polarization along the propagation of the major energy streamline describes a complex evolution, as shown in the bottom panel of Fig.5(b). One interpretation can be that none of the symmetry operations link the successive vortices guiding these streamlines.

The situation is rather different for the bonding modes. It can be noted that the vortices organize the local currents of energy so that the total flow is null to respect the ZGV. In addition to the spiral currents, the energy fluxes in the $\mathrm{PhC}$ bulks and inside the waveguide are propagating in opposite directions. If the major streamline is also circulating between vortices with opposite topological charges, as shown in the middle panels of Fig.5(c)-(d), the optical vortices are not located near the PhC parts but rather inside the waveguide. It is immediately apparent that the phase structure of the $\mathrm{TE}_{1}^{+}$mode has a strong phase modulation extended all over the structure with phase gradients forcing one vortex to bond with three vortices characterized by opposite topological charges. The phase pattern of this mode takes the form of shrunken hexagons reminiscent of the structural arrangement of graphene. Regarding the $\mathrm{TE}_{1}^{-}$mode, because the phase modulation is constrained within the core of the waveguide, the vortices only bond through the glide-plane symmetry axes thereby forming a chain similar to poly-acetylene. These similarities with organic chemistry come from the triangular lattice, suggesting that other singularities arrangements may be formed by choosing another crystal lattice.

The optical vortices present in the field pattern of these modes play a completely different role with regards to the evolution of the polarization along the major streamline. It is well-known that vortex beams in free-space like Laguerre-Gaussian beams with higher order possess longitudinal OAM around which the polarization rotates in the $S_{1} S_{2}$-plane[13,14]. This means that the parameter $2 \alpha$ is periodically evolving over $2 \pi c / \lambda$ in these beams. In our case, if the net orbital momentum of the PCW modes is null, the transverse aspects of the optical vortices seem to contribute in a different way to the motion of the polarization state on the Poincaré sphere. For both the $\mathrm{TE}_{1}^{+}$ and $\mathrm{TE}_{1}^{-}$modes, their polarization vectors rotate in the $S_{2} S_{3}$-plane around a constant $S_{1}$ value, as shown in the bottom panels of Fig.5(c)-(d). The difference in their $S_{1}$ values is associated with the spanning of the vortices in the $y$ direction. For the $\mathrm{TE}_{1}^{+}$mode, their proximity limits the swinging amplitude of the energy flow. Simultaneously, the degree of ellipticity is shortly decreasing outward of the C-points. In the case of the $\mathrm{TE}_{1}^{-}$mode, the ellipticity remains important in the transverse direction outward of the SAM locations while the major energy streamline smoothly turns around the vortices. In both cases, their polarization vectors turn in the anti-clockwise direction but with a $\pi$-shift difference: the polarization of the $\mathrm{TE}_{1}^{+}\left(\mathrm{TE}_{1}^{-}\right)$mode is described by $2 \alpha=\pi / 2(-\pi / 2)$ at the border of the unit-cell (c.f. bottom panel of Fig.4.b). This evolution of the polarization along the energy flux is 


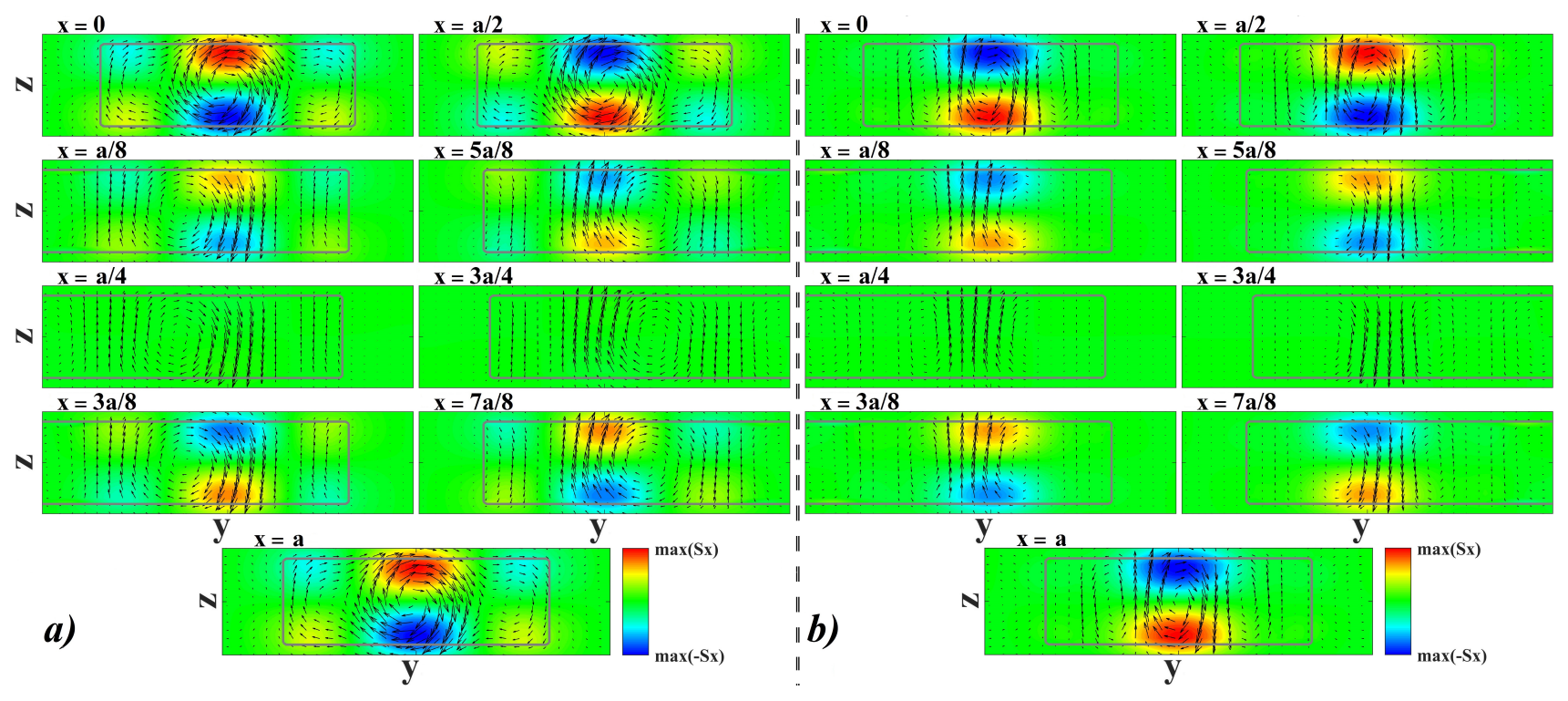

FIG. 6. SAM density of the $\mathrm{TE}_{1}^{+}$(a) and $\mathrm{TE}_{1}^{-}$(b) modes along the propagation direction at different x positions in the unit-cell. The arrows describe the components $S_{y}$ and $S z$ transverse to the propagation while the colored plot represents the longitudinal component $S_{x}$.

analogous to spin waves[47] and thus can be described by a modified Landau-Lifshitz equation of motion without damping[48]:

$$
\frac{d}{d t}\left(\begin{array}{l}
S_{1} \\
S_{2} \\
S_{3}
\end{array}\right) \frac{1}{S_{0}}=-\frac{2 \pi c}{\lambda}\left(\begin{array}{l}
S_{1} \\
S_{2} \\
S_{3}
\end{array}\right) \frac{1}{S_{0}} \times \overrightarrow{P_{e f f}},
$$

where $\overrightarrow{P_{\text {eff }}}$ is an effective polarization driving the precession of the polarization state. Because, the analysis is conducted on the streamlines guided by the optical vortices, a natural choice would be the polarization state at the center of the vortices. Of course, the conservation of the global chirality ensured by the mirror symmetry in the symmorphic $\mathrm{PCW}$ is guaranteed by the glide-plane symmetries for $m \neq 0$ :

$$
S_{3}(x, y)=-S_{3}(x+m,-y)=-S_{3}(x+m-a,-y),
$$

a relation which links the polarization in the center of the vortices in question. Therefore, the relevant Stokes parameter to consider is the $S_{1}$ component. Surprisingly, the projected electric field is oriented there along the $y$ axis for both the $\mathrm{TE}_{1}^{+}$and $\mathrm{TE}_{1}^{-}$modes $(2 \alpha=\pi)$ and thus makes Eq.(10) absolutely valid by setting $\overrightarrow{P_{\text {eff }}}=$ $(-1,0,0)$. This dynamic reveals that the alternative transverse OAM progressively invert the SAM through the glide-plane symmetry in order to conserve the global chirality of the modes without annihilating the local chirality as it does at the band-edge. The other energy streamlines trapped between the optical vortices are also forming circular contours on the Poincare sphere surface but their effective polarizations have a non-zero $2 \omega$ value. Being an affair of polarization, a qualitative description of these streamlines by Eq.(10) remains true as the ellipticity of the effective polarization vector is in line with the proximity of one or another vortex. However, a quantitative picture must take into account an appropriate charge potential decaying with the relative distance of the streamlines with the OAM.

\section{AM DENSITIES AND SPIN-ORBIT COUPLING}

The previous section allows the clear identification of the geometrical phases present in both the electric and magnetic fields profiles of the bonding modes, it was also noted that the optical vortices influence the state of polarization along the energy streamlines. Nevertheless, this study has been so far restricted to the plane of symmetry of the PCW while a three-dimensional analysis of the modes is required to unveil the true interplay between the spin and orbital degrees of freedom, especially where electric and magnetic fields are not orthogonal and so the field is partially polarized $[1,45,46]$. Recent interests about optical momenta, including photonic wheels, lead towards a deeper understanding of the dynamics of wavefields in inhomogeneous media. The main interest to study these characteristics lies in their canonical aspects, they enable the description of the structured field beyond its kinetic picture. For example, these considerations yield to the demonstration of the conservation and quantization of the total AM number for modes confined in cylindrical fibers and metallic wires[30]. Moreover, taking into account dispersive effects discloses the transverse character of the spin of a surface plasmon-polariton at a metal-vacuum interface, which is equal in absolute value to the electron spin contribution per plasmon at its maximum frequency[29]. 
Nevertheless, to the best of our knowledge, the local currents of spin and orbitals AM have not been explored in PhCs yet. Only the SAM density has been described in the plane of symmetry to explore the non-zero chirality of a PCW mode trapped between different $\mathrm{PhCs}$ with trivial and non-trivial photonic bandgaps[49], thus ignoring its vectorial nature. Defining both the SAM and OAM densities across all the PhC slab reveals interesting properties of the optical momenta brought by both the photonic bandgap and the total internal reflection. Accordingly to the developed formalism based on the Minkowski description of the light[29, 30, 44], the linear (canonical) momentum, spin and orbital AM densities can be expressed in our system as:

$$
\begin{array}{r}
\vec{P}=\frac{\lambda}{8 \pi c} \operatorname{Im}\left(\epsilon \mathrm{E}^{*} \cdot \vec{\nabla}(\overrightarrow{\mathrm{E}})+\mu \overrightarrow{\mathrm{H}}^{*} \cdot \vec{\nabla}(\overrightarrow{\mathrm{H}})\right), \\
\vec{S}=\frac{\lambda}{8 \pi c} \operatorname{Im}\left(\epsilon \overrightarrow{\mathrm{E}}^{*} \times \overrightarrow{\mathrm{E}}+\mu \overrightarrow{\mathrm{H}}^{*} \times \overrightarrow{\mathrm{H}}\right), \\
\vec{L}=\vec{r} \times \vec{P},
\end{array}
$$

where $\epsilon$ is the permittivity and $\mu$ is the permeability of the dielectrics. In our case, the dimensions of the structure set the frequencies of the modes in the telecom bandwidth which allows any dispersion correction for both air and Si to be neglected. As mentioned before, any geometrical phase vanishes at the band-edge because the fields envelops are purely real at the edge of the BZ and the same applies for the AM densities. Therefore, it is not the appropriate wave-vector to study any coaction between slow-light and AM because they compete against each other, although both of them are supposed to be strengthened by the tight confinement of light. However, in our system, it has been shown in the former section that the alternative opposite transverse OAM trap nonvanishing spiral energy fluxes to satisfy the ZGV inside the BZ, so it stands to reason that the analysis of the local flows of $\mathrm{AM}$ densities must provide a deeper insight into the nature of photonic wheels at ZGV.

\section{A. SAM Density}

Since the SAM density is by definition normal to the polarization ellipse, it is limited to the electric field contribution only in the plane of symmetry. Furthermore, restricting the analysis of the SAM density to this plane conceals the local currents in the $x$ and $y$ directions. For instance, despite that the PCW modes are locally chiral, as seen previously, their helicity density $\left(\propto \operatorname{Im}\left(\overrightarrow{\mathrm{E}} \cdot \vec{H}^{*}\right)\right)$ there is identically zero. On the other hand, the modes are guided by wave-vectors parallel to the $x$-axis in the global sense, which prompt the examination of the SAM density behavior along the direction of propagation.

Figures 6(a) and (b) show the SAM density in the $y z$ plane at different $\mathrm{x}$ positions in the unit-cell for both the $\mathrm{TE}_{1}^{+}$and $\mathrm{TE}_{1}^{-}$modes respectively. The arrows represent the projection of the SAM density in the $y z$-plane
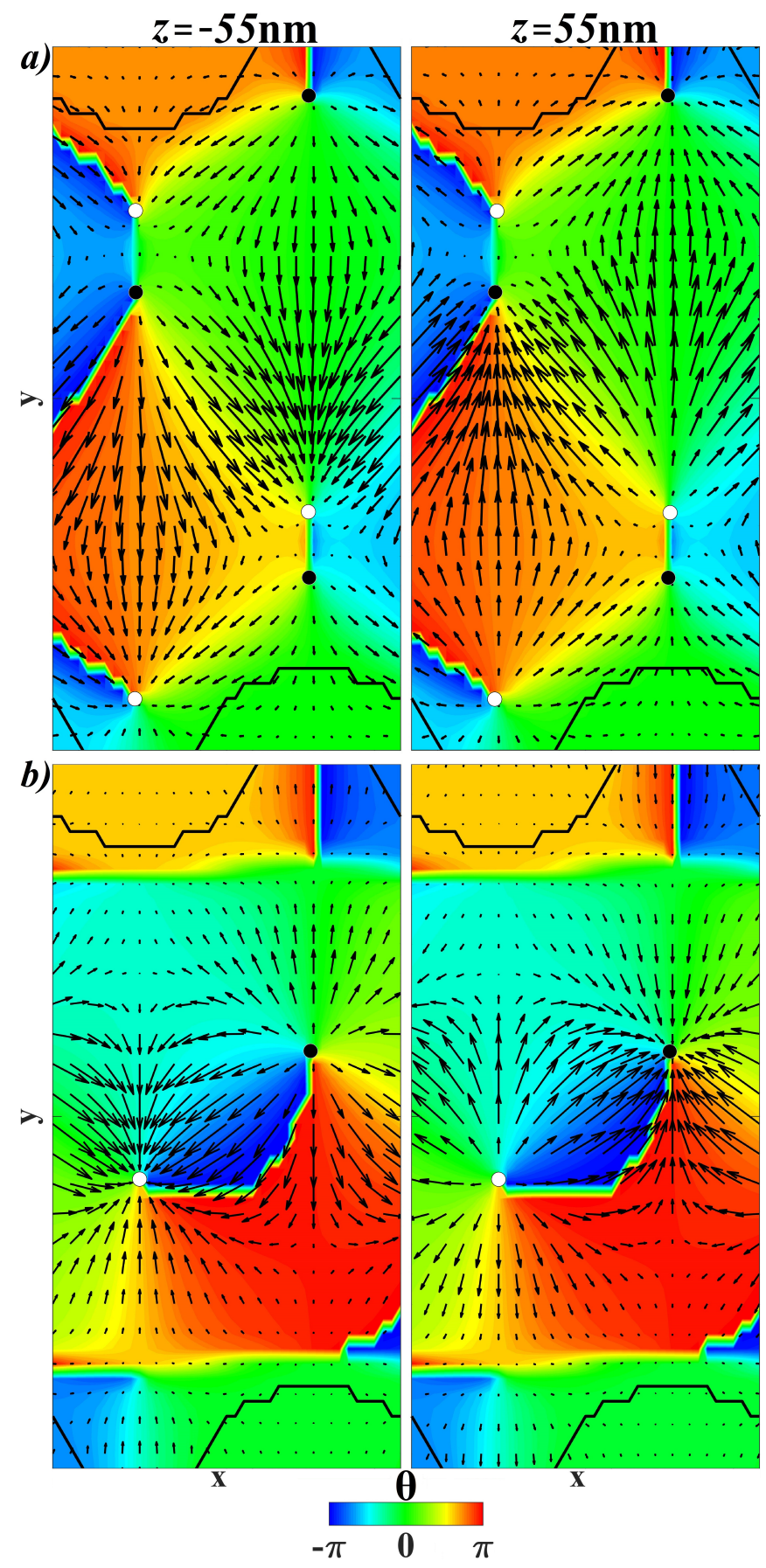

FIG. 7. The SAM density projected on the $x y$-plane at $\mathrm{z}= \pm t / 4$ for the $\mathrm{TE}_{1}^{+}$(a) and $\mathrm{TE}_{1}^{-}$(b) modes. The projection is represented by the black arrows, the phase pattern of the magnetic fields $\theta$ by the colored plot and topological charges $l= \pm 1$ by white and black circles respectively.

while the colored contour plot represents the longitudinal component. The striking feature of the transverse components is the emergence of vortices in the PCW to link the flow exclusively oriented along the $z$-axis in the plane of symmetry. If the vorticity is conserved by the glideplane axes, it can be noted that the longitudinal compo- 
nent is odd with respect to both the glide-plane axes and the plane of symmetry, thus ensuring the conservation of the chirality. This organization of the SAM density flows translates its vortex character in the $x$ direction as well, trapping non-vanishing flux inside the dielectric. These symmetry relations under glide-plane operations reflect the presence of sources and drains for the SAM density, which is supported by the fact that the longitudinal component is null at $\mathrm{x}=a / 4$ and $3 a / 4$, positions that coincide with the locations of both the C-points and the centers of the vortices.

In order to examine the structure of the local currents from another perspective, the SAM density is represented by arrows in the $x y$-plane at different heights $(z= \pm t / 4)$ for both the $\mathrm{TE}_{1}^{+}$and $\mathrm{TE}_{1}^{-}$modes in Fig.7(a) and (b) respectively and is superimposed on the $\theta$ pattern of the modes at $z=0$. This choice to overlap the SAM density with the plot that is revealing the OAM has been deliberately made to bring to light the spinorbit coupling. The calculation shows that the topological charges $l$ generate SAM density fluxes which leads towards vortices with opposite topological charges. In other words, optical vortices are both sources and drains of the SAM density and their topological charges determine the sign of its direction along the $z$-axis. The superimposition also discloses that the streamlines of $\vec{S}$ follow constant $\theta$ values such as SAM circulations cannot be established between OAM with same topological charge. Pieced together, these characteristics draw a clear picture of the SAM density circulation: the bonding modes can be compared at different arrangements of spins oriented in the opposite direction of their first neighbors, confirming the anti-ferromagnets analogy developed in the former section. It is also in agreement with the Ising picture of anti-ferromagnetic materials[50] (a negative exchange constant between the first neighbor spins). For the $\mathrm{TE}_{1}^{+}$mode, topological charges bond with three opposite topological charges while only with two for the $\mathrm{TE}_{1}^{-}$mode, thus qualitatively explaining why they are the lower and upper energy levels respectively.

\section{B. Linear Momentum and OAM Densities}

The linear momentum density is an interesting property quantifying the propagation direction of the electric and magnetic fields and can be interpreted as a local wave-vector of the modes. The non-paraxial guidance of the fields in any $\mathrm{PhC}$ system requires the consideration of both fields contribution in order to properly define the linear momentum and the AM densities. However, the mode confinement in a slab much thinner than the wavelength, as it is the case here, implies that the fields remain mostly TE by continuity[1]. Accordingly with its expression and this consideration, $\vec{P}$ is essentially pointing in the $x y$-plane inside the PCW. The modes are considered in the special framework of ZGV which prohibits the overall linear momentum flow along the propagation direction because the fields carry the electromagnetic energy. The linear momentum density in the $x y$ plane is split into electric and magnetic parts represented in Fig.8(a) and (b) for the $\mathrm{TE}_{1}^{+}$and $\mathrm{TE}_{1}^{-}$modes respectively and superimposed on the relevant energy density. Similarly, the electric field part of the linear momentum density can be decomposed into a LCP, a RCP and an out-of-plane components which allows for a separate representation as:

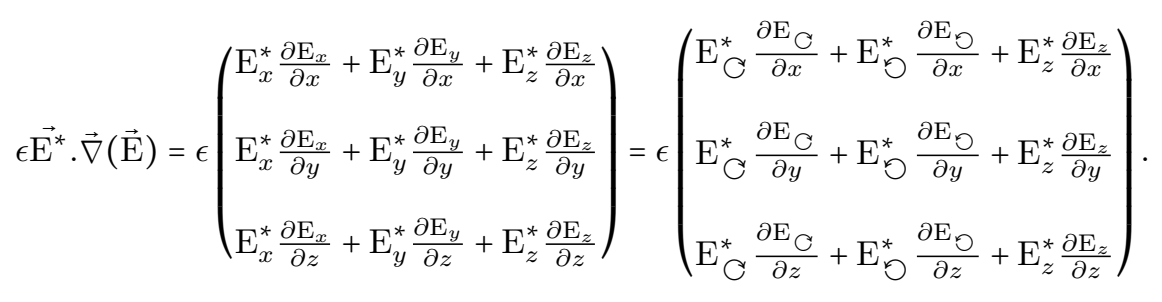

It can be noted that by comparing the left panels of Fig.(8), the energy density of the LCP and RCP components are not only spatially distinct, because the longitudinal and transverse components are overlapping with $\delta=\pi / 2$, but also related by the glide-plane symmetry as well as their associated linear momentum densities such as:

$$
\begin{aligned}
& \epsilon\left|\overrightarrow{\mathrm{E}}_{\wp}\right|^{2}(x, y, z)=\epsilon\left|\overrightarrow{\mathrm{E}}_{\mathrm{C}}\right|^{2}(x+m,-y, z),
\end{aligned}
$$

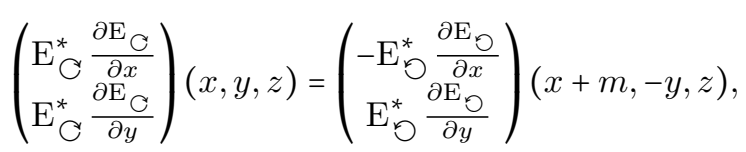

thus establishing the circular polarization as an appropriate basis to characterize these PCW modes with enhanced chirality. It is also convenient to localize the LCP and RCP flows and outline the path guiding the 

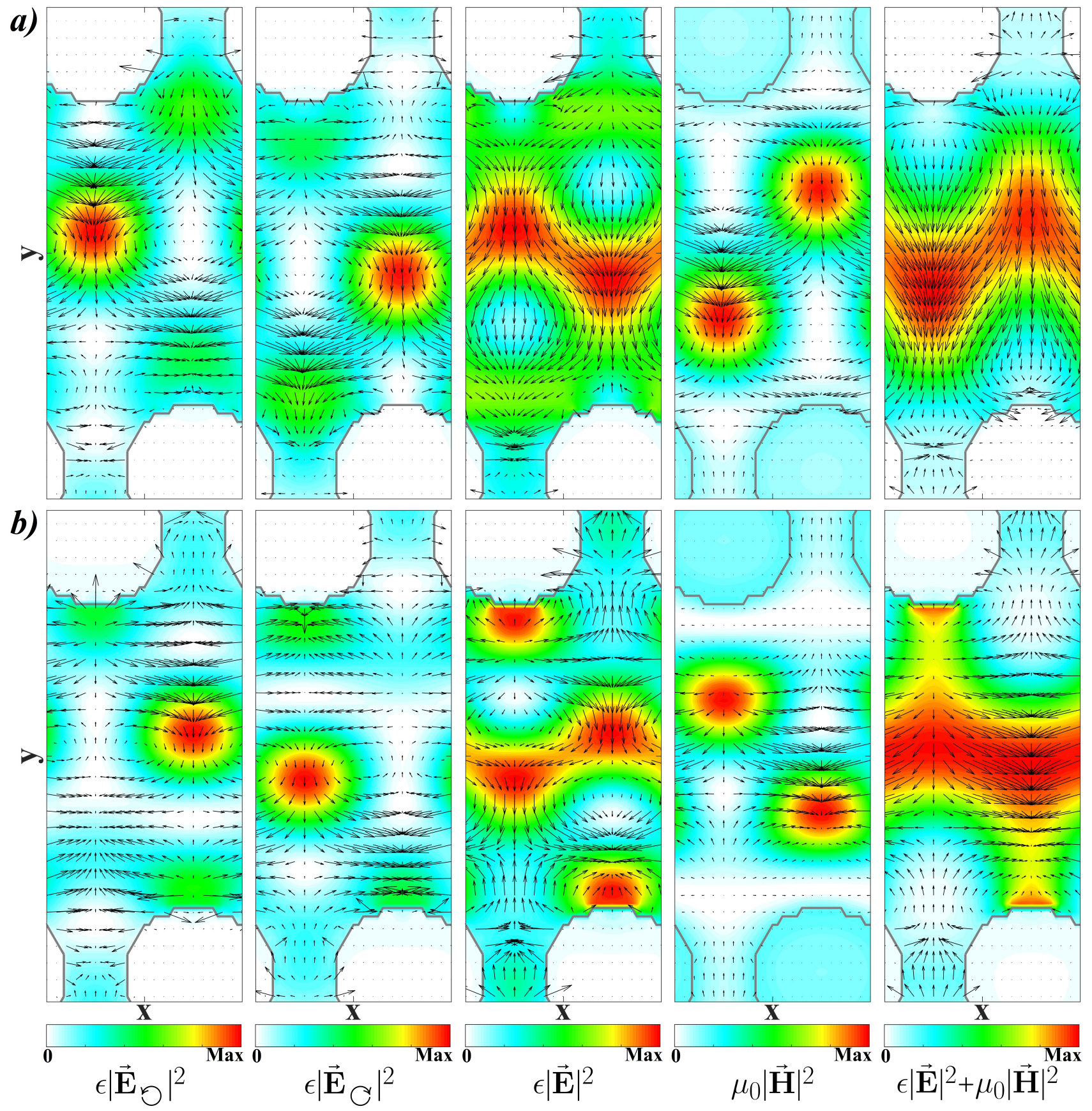

FIG. 8. The linear $\mathrm{AM}$ density components of the $\mathrm{TE}_{1}^{+}$(a) and $\mathrm{TE}_{1}^{-}$(b) modes represented by black arrows and superimposed on the energy density profiles from left to right: the LCP component of the electric field, the RCP component of the electric field, the total electric field, the magnetic field and the total electromagnetic fields.

linear AM density together. Equation (14) establishes that the linear momentum density does not propagate along the propagation direction because opposite local currents obeying the mirror symmetry at $x=a / 4$ and $3 a / 4[39]$ annihilate the total flows. Nevertheless, two distinct manners to satisfy the ZGV are observable in the linear momentum densities of the $\mathrm{TE}_{1}^{+}$and $\mathrm{TE}_{1}^{-}$modes. If the linear density of the $\mathrm{TE}_{1}^{+}$mode is not propagating due to clear local drifts of the fields in the $y$ direction, the $\mathrm{TE}_{1}^{-}$mode shows clear opposite local currents pointing along the $x$-axis that are emerging from $x=a / 4$ and merging to $x=3 a / 4$ in each unit-cell.

The OAM density represents the orbital motion of light, it is therefore locally normal to the orbital plane of the fields. Despite the facts that the linear momentum is highly confined in the Si region and its $z$ component is negligible, an appropriate frame of reference must be established because this quantity is a torque. As ex- 
plained before, the modes are associated with a global wave-vector pointing along $\vec{x}$ so the OAM density needs to be defined in the $y z$-plane along the $x$-axis. On the other hand, the origin of the torque in this plane is nontrivial due to the lack of mirror symmetry, a suitable origin is the energy barycenter which has its $y$ coordinate defined by :

$$
y_{0}(x)=\frac{\int_{-\infty}^{\infty} y\left(\mu_{0}|\overrightarrow{\mathrm{H}}(x, y, z)|^{2}+\epsilon|\overrightarrow{\mathrm{E}}(x, y, z)|^{2}\right) d z d y}{\int_{-\infty}^{\infty}\left(\mu_{0}|\overrightarrow{\mathrm{H}}(x, y, z)|^{2}+\epsilon|\overrightarrow{\mathrm{E}}(x, y, z)|^{2}\right) d z d y} .
$$

Due to the symmetry along the slab thickness, the $z$ coordinate of the energy barycenter is simply $z_{0}=0$. If the OAM density also presents a vortex structure in the $y z$-plane, its vorticity changes sign along the propagation while its longitudinal component is even with respect to the glide-plane symmetry, the opposite relations when compared with the SAM density (Fig.6). To compare the behavior of the OAM density with respect to the spin of light, the former is plotted in the $x z$-plane at y positions containing the C-points in Fig.9(a) and (b) for the $\mathrm{TE}_{1}^{+}$ and $\mathrm{TE}_{1}^{-}$modes respectively, and are superimposed onto the Stokes parameter $S_{3}$. The landscapes demonstrate that the OAM is uniformly transverse in the plane of symmetry and emphasize how the OAM density fluxes allow optical vortices with opposite topological charges to coexist in the unit-cell. It appears that the OAM density is rotating in this plane so that the C-points are located at the tips between two vortices centered in neighboring unit-cells. In other words, the rotation of the orbital motion of light changes direction across C-point locations, besides changing the direction of rotation as well between C-points, at the center of the OAM density vortices. Furthermore, this configuration highlights that the OAM circulating fluxes are trapped between Cpoints with the same handedness with high field intensity. Despite the close proximity of the C-points with opposite handedness in the $y$ direction, the vortex structure of the OAM density abruptly changes to confine spiral flows accordingly. It is also in agreement with the magnetic fields profiles holding alternative OAM shaping the undulated flows of energy of the modes, bringing into view another aspect of the spin-orbit coupling occurring in this PCW. The rotation of the OAM density at the C-point locations support optical vortices with opposite topological charges to exist simultaneously in the same unit-cell.

\section{CONCLUSION}

In summary, it has been shown that breaking the PCW parity can lead to the formation of hybrid modes supporting both enhanced transverse SAM and OAM at anomalous ZGV points. Clear interplays between these two degrees of freedom are noted throughout the paper. Along the major energy streamlines, the motion of the polarization on the Poincaré sphere is analogous to helical spin waves and can be described by a modified

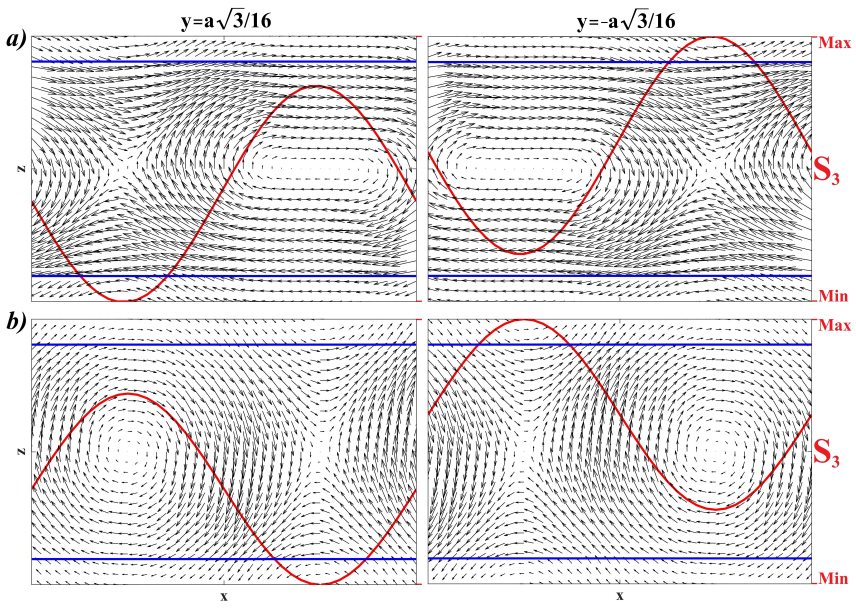

FIG. 9. The OAM density of the $\mathrm{TE}_{1}^{+}$(a) and $\mathrm{TE}_{1}^{-}$(b) modes in the $x z$-plane represented by black arrows and superimposed on the Stokes Parameter $S_{3}(\mathrm{x}, \mathrm{y}, 0)$ plotted in red for $y= \pm a \sqrt{3} / 16$. The structure is outlined by blue lines.

Landau-Lifshitz equation where the optical vortices play the role of an effective polarization vector. Moreover, the AM densities have been investigated all across the PCW structure in the particular context of the very slow-light regime and reveal a peculiar behaviour between the spin and orbital parts of the AM. The SAM density has been found to be generated by the optical vortices bonding them like an anti-ferromagnetic material while the OAM density forms spiral flows trapped between C-points.

Moreover, the detailed study of the AM flows in three dimensions allows one to understand how the spin-orbit coupling can be engineered in subwavelength-based devices like $\mathrm{PhCs}$ by the means of structural modifications with possible applications such as single nano-particle sensors with the potential to identify their chirality[25], optical tweezers $[51,52]$ and unidirectional emission of embedded quantum emitters [27, 28]. In addition, this coupling between spin and orbital parts of the AM also opens the perspective for controlling one degree of freedom with the other and inducing spin to orbital conversion of light and vice-versa[34, 53, 54], engineer directional routing of light on-chip as well as the spinHall effect of light in cleverly tailored nano-photonic components[31]. Moreover, the break of other symmetries can lead to the emergence of a net AM that can be transmitted through the PCW, for example a waveguide at the interface between two $\mathrm{PhCs}$ with different topologies[49]. There is also potential to engineer the rotation of the OAM; i.e. from longitudinal to transverse or vice-versa, by adiabatically interfacing such a PCW holding photonic wheels with a trench waveguide supporting longitudinal OAM[55] modes. Last but not least, other numerous applications can take advantage of the slow-light regime such as non-linear photonics $[6,7]$ with the benefit of combining it with spin or orbital selection rules. 
The data from the paper can be obtained from the University of Southampton ePrint research repository: https://doi.org/10.5258/SOTON/D0760

\section{ACKNOWLEDGEMENT}

This work was funded by an Engineering and Physical Sciences Research Council (EPSRC) grant
(EP/M009416/1, EP/M008975/1), an EU FP7 People: Marie-Curie Actions (PEOPLE) grant (PCIG13GA2013-618116) and the University of Southampton Zepler Institute Research Collaboration Stimulus Fund. M.S. and S.S gratefully acknowledges Prof. Harvey Rutt and Daniel Burt for fruitful discussions.
[1] John D Joannopoulos, Steven G Johnson, Joshua N Winn, and Robert D Meade. Photonic crystals: molding the flow of light. Princeton university press, 2011.

[2] Steven G Johnson, Shanhui Fan, Pierre R Villeneuve, John D Joannopoulos, and LA Kolodziejski. Guided modes in photonic crystal slabs. Physical Review B, 60 (8):5751, 1999.

[3] Eli Yablonovitch. Inhibited spontaneous emission in solid-state physics and electronics. Physical review letters, 58(20):2059, 1987.

[4] Alvaro Blanco, Emmanuel Chomski, Serguei Grabtchak, Marta Ibisate, Sajeev John, Stephen W Leonard, Cefe Lopez, Francisco Meseguer, Hernan Miguez, Jessica P Mondia, et al. Large-scale synthesis of a silicon photonic crystal with a complete three-dimensional bandgap near 1.5 micrometres. Nature, 405(6785):437, 2000.

[5] Susumu Noda, Katsuhiro Tomoda, Noritsugu Yamamoto, and Alongkarn Chutinan. Full threedimensional photonic bandgap crystals at near-infrared wavelengths. Science, 289(5479):604-606, 2000.

[6] Toshihiko Baba. Slow light in photonic crystals. Nature photonics, 2(8):465, 2008.

[7] Thomas F Krauss. Why do we need slow light? Nature Photonics, 2(8):448, 2008.

[8] Yurii A Vlasov, Martin O'boyle, Hendrik F Hamann, and Sharee J McNab. Active control of slow light on a chip with photonic crystal waveguides. Nature, 438(7064):65, 2005.

[9] Masaya Notomi, K Yamada, Akihiko Shinya, J Takahashi, C Takahashi, and I Yokohama. Extremely large group-velocity dispersion of line-defect waveguides in photonic crystal slabs. Physical Review Letters, 87(25): 253902, 2001.

[10] VSC Manga Rao and Stephen Hughes. Single quantumdot purcell factor and $\beta$ factor in a photonic crystal waveguide. Physical Review B, 75(20):205437, 2007.

[11] Stephen Hughes. Enhanced single-photon emission from quantum dots in photonic crystal waveguides and nanocavities. Optics letters, 29(22):2659-2661, 2004.

[12] Marta Arcari, Immo Söllner, Alisa Javadi, S Lindskov Hansen, Sahand Mahmoodian, Jin Liu, Henri Thyrrestrup, Eun Hye Lee, Jin Dong Song, Søren Stobbe, et al. Near-unity coupling efficiency of a quantum emitter to a photonic crystal waveguide. Physical review letters, 113(9):093603, 2014.

[13] Giovanni Milione, HI Sztul, DA Nolan, and RR Alfano. Higher-order poincaré sphere, stokes parameters, and the angular momentum of light. Physical review letters, 107 (5):053601, 2011.
[14] Giovanni Milione, S Evans, DA Nolan, and RR Alfano. Higher order pancharatnam-berry phase and the angular momentum of light. Physical review letters, 108(19): 190401, 2012

[15] Andrey A Sukhorukov, Sangwoo Ha, Anton S Desyatnikov, Andrei V Lavrinenko, and Yuri S Kivshar. Slowlight vortices in periodic waveguides. Journal of Optics A: Pure and Applied Optics, 11(9):094016, 2009.

[16] Matteo Burresi, RJP Engelen, Aron Opheij, Dries Van Oosten, Daisuke Mori, Toshihiko Baba, and L Kuipers. Observation of polarization singularities at the nanoscale. Physical review letters, 102(3):033902, 2009.

[17] Andrew B Young, ACT Thijssen, Daryl M Beggs, Petros Androvitsaneas, L Kuipers, John G Rarity, Stephen Hughes, and Ruth Oulton. Polarization engineering in photonic crystal waveguides for spin-photon entanglers. Physical review letters, 115(15):153901, 2015.

[18] Peter Banzer, Martin Neugebauer, Andrea Aiello, Christoph Marquardt, Norbert Lindlein, Thomas Bauer, and Gerd Leuchs. The photonic wheel: demonstration of a state of light with purely transverse angular momentum. arXiv preprint arXiv:1210.1772, 2012.

[19] Andrea Aiello and Peter Banzer. The ubiquitous photonic wheel. Journal of Optics, 18(8):085605, 2016.

[20] Andrea Aiello, Peter Banzer, Martin Neugebauer, and Gerd Leuchs. From transverse angular momentum to photonic wheels. Nature Photonics, 9(12):789, 2015.

[21] Martin Neugebauer, Thomas Bauer, Andrea Aiello, and Peter Banzer. Measuring the transverse spin density of light. Physical review letters, 114(6):063901, 2015.

[22] Konstantin Y Bliokh, Aleksandr Y Bekshaev, and Franco Nori. Extraordinary momentum and spin in evanescent waves. Nature communications, 5:3300, 2014.

[23] Konstantin Y Bliokh and Franco Nori. Transverse and longitudinal angular momenta of light. Physics Reports, 592:1-38, 2015.

[24] Francisco J Rodríguez-Fortuño, Giuseppe Marino, Pavel Ginzburg, Daniel OConnor, Alejandro Martínez, Gregory A Wurtz, and Anatoly V Zayats. Near-field interference for the unidirectional excitation of electromagnetic guided modes. Science, 340(6130):328-330, 2013.

[25] Francisco J Rodríguez-Fortuño, Isaac Barber-Sanz, Daniel Puerto, Amadeu Griol, and Alejandro Martínez. Resolving light handedness with an on-chip silicon microdisk. ACS Photonics, 1(9):762-767, 2014.

[26] Christian Junge, Danny Oshea, Jürgen Volz, and Arno Rauschenbeutel. Strong coupling between single atoms and nontransversal photons. Physical review letters, 110 
(21):213604, 2013.

[27] Immo Söllner, Sahand Mahmoodian, Sofie Lindskov Hansen, Leonardo Midolo, Alisa Javadi, Gabija Kiršanskè, Tommaso Pregnolato, Haitham El-Ella, Eun Hye Lee, Jin Dong Song, et al. Deterministic photon-emitter coupling in chiral photonic circuits. $\mathrm{Na}$ ture nanotechnology, 10(9):nnano-2015, 2015.

[28] Sabyasachi Barik, Aziz Karasahin, Christopher Flower, Tao Cai, Hirokazu Miyake, Wade DeGottardi, Mohammad Hafezi, and Edo Waks. A topological quantum optics interface. Science, 359(6376):666-668, 2018.

[29] Konstantin Y Bliokh, Aleksandr Y Bekshaev, and Franco Nori. Optical momentum and angular momentum in complex media: from the abraham-minkowski debate to unusual properties of surface plasmon-polaritons. New Journal of Physics, 19(12):123014, 2017.

[30] MF Picardi, KY Bliokh, FJ Rodríguez-Fortuño, F Alpeggiani, and F Nori. Angular momenta, helicity, and other properties of dielectric-fiber and metallic-wire modes. arXiv preprint arXiv:1805.03820, 2018.

[31] Masaru Onoda, Shuichi Murakami, and Naoto Nagaosa. Hall effect of light. Physical review letters, 93(8):083901, 2004.

[32] Tzuhsuan Ma and Gennady Shvets. All-si valley-hall photonic topological insulator. New Journal of Physics, 18(2):025012, 2016.

[33] Xiao-Dong Chen, Fu-Li Zhao, Min Chen, and Jian-Wen Dong. Valley-contrasting physics in all-dielectric photonic crystals: orbital angular momentum and topological propagation. Physical Review B, 96(2):020202, 2017.

[34] Jian-Long Liu, Wei-Min Ye, and Shuang Zhang. Pseudospin-induced chirality with staggered optical graphene. Light: Science \& Applications, 5(8):e16094, 2016.

[35] Sabyasachi Barik, Hirokazu Miyake, Wade DeGottardi, Edo Waks, and Mohammad Hafezi. Two-dimensionally confined topological edge states in photonic crystals. New Journal of Physics, 18(11):113013, 2016.

[36] Long-Hua $\mathrm{Wu}$ and Xiao $\mathrm{Hu}$. Scheme for achieving a topological photonic crystal by using dielectric material. Physical review letters, 114(22):223901, 2015.

[37] A Yu Petrov and M Eich. Zero dispersion at small group velocities in photonic crystal waveguides. Applied Physics Letters, 85(21):4866-4868, 2004.

[38] Moïse Sotto, Kapil Debnath, Ali Z Khokhar, Isao Tomita, David Thomson, and Shinichi Saito. Anomalous zerogroup-velocity photonic bonding states with local chirality. JOSA B, 35(10):2356-2363, 2018.

[39] Adam Mock, Ling Lu, and John OBrien. Space group theory and fourier space analysis of two-dimensional photonic crystal waveguides. Physical Review B, 81(15): $155115,2010$.

[40] Moise Sotto, Sala Henri, Isao Tomita, Kapil Debnath, and Shinichi Saito. Polarisation rotation and mode splitting in photonic crystal line-defect waveguides. Frontiers in Materials, 2018.

[41] Gene Dresselhaus. Spin-orbit coupling effects in zinc blende structures. Physical Review, 100(2):580, 1955.

[42] M Ibanescu, SG Johnson, D Roundy, C Luo, Y Fink, and JD Joannopoulos. Anomalous dispersion relations by symmetry breaking in axially uniform waveguides. Physical review letters, 92(6):063903, 2004.

[43] Mihai Ibanescu, Steven G Johnson, David Roundy, Yoel Fink, and JD Joannopoulos. Microcavity confinement based on an anomalous zero group-velocity waveguide mode. Optics letters, 30(5):552-554, 2005.

[44] Konstantin Y Bliokh, Aleksandr Y Bekshaev, and Franco Nori. Optical momentum, spin, and angular momentum in dispersive media. Physical review letters, 119(7): 073901, 2017.

[45] Mark R Dennis. Fermionic out-of-plane structure of polarization singularities. Optics letters, 36(19):3765-3767, 2011.

[46] Zhi-Cheng Ren, Ling-Jun Kong, Si-Min Li, Sheng-Xia Qian, Yongnan Li, Chenghou Tu, and Hui-Tian Wang. Generalized poincare sphere. Optics express, 23(20): 26586-26595, 2015.

[47] F Duncan M Haldane. Nonlinear field theory of largespin heisenberg antiferromagnets: semiclassically quantized solitons of the one-dimensional easy-axis néel state. Physical Review Letters, 50(15):1153, 1983.

[48] Ya B Bazaliy, BA Jones, and Shou-Cheng Zhang. Modification of the landau-lifshitz equation in the presence of a spin-polarized current in colossal-and giantmagnetoresistive materials. Physical Review B, 57(6): R3213, 1998.

[49] Wei-Min Deng, Xiao-Dong Chen, Fu-Li Zhao, and JianWen Dong. Transverse angular momentum in topological photonic crystals. Journal of Optics, 20(1):014006, 2017.

[50] Roy J Glauber. Time-dependent statistics of the ising model. Journal of mathematical physics, 4(2):294-307, 1963.

[51] V Garcés-Chávez, D McGloin, MJ Padgett, W Dultz, H Schmitzer, and K Dholakia. Observation of the transfer of the local angular momentum density of a multiringed light beam to an optically trapped particle. Physical review letters, 91(9):093602, 2003.

[52] AT O'neil, I MacVicar, L Allen, and MJ Padgett. Intrinsic and extrinsic nature of the orbital angular momentum of a light beam. Physical review letters, 88(5):053601, 2002.

[53] Konstantin Y Bliokh, Elena A Ostrovskaya, Miguel A Alonso, Oscar G Rodríguez-Herrera, David Lara, and Chris Dainty. Spin-to-orbital angular momentum conversion in focusing, scattering, and imaging systems. Optics express, 19(27):26132-26149, 2011.

[54] Konstantin Y Bliokh, Miguel A Alonso, Elena A Ostrovskaya, and Andrea Aiello. Angular momenta and spinorbit interaction of nonparaxial light in free space. Physical Review A, 82(6):063825, 2010.

[55] Shuang Zheng and Jian Wang. On-chip orbital angular momentum modes generator and (de) multiplexer based on trench silicon waveguides. Optics express, 25(15): 18492-18501, 2017. 